\title{
The new perspectives of targeted therapy in acute myeloid leukemia
}

\author{
Angela Walasek ${ }^{A-F}$ \\ Department and Clinic of Neoplasms and Bone Marrow Transplantation, Wroclaw Medical University, Poland \\ A - research concept and design; B - collection and/or assembly of data; $C$ - data analysis and interpretation; \\ $\mathrm{D}$ - writing the article; $\mathrm{E}$ - critical revision of the article; $\mathrm{F}$ - final approval of the article
}

\section{Address for correspondence}

Angela Walasek

E-mail: angela.walasek@gmail.com

\section{Funding sources \\ None declared}

Conflict of interest

None declared

Received on November 27, 2017

Reviewed on December 12, 2017

Accepted on December 28, 2017

Published online on August 24, 2018

\begin{abstract}
Acute myeloid leukemia (AML) is a heterogeneous disease and the results of previous treatment with cytotoxic drugs have not been satisfactory. This situation has prompted investigations into novel approaches. The breakthrough in therapy brought by all-trans retinoic acid (ATRA) in acute promyelocytic leukemia (APL) and tyrosine kinase inhibitors in neoplasms with the Philadelphia chromosome has encouraged the search for other effective targeted therapies. Among the tested substances are higher molecular mass drugs such as antibodies and various small molecules: kinase inhibitors, cell pathway inhibitors and epigenetic modulators. So far, the U.S. Food and Drug Administration (FDA) has approved the antibody-drug conjugate gemtuzumab ozogamycin (GO), the tyrosine kinase inhibitor midostaurin and the IDH2 inhibitor enasidenib. These studies have led to a better understanding of the mechanisms of leukemogenesis and may soon allow for differentiating treatments depending on baseline mutational complements. Some innovative drugs described in this article have strong therapeutic potential, but there is still a long way to go before actual success in targeted treatment.
\end{abstract}

Key words: immunotherapy, target therapy, acute myeloid leukemia

Cite as

Walasek A. The new perspectives of targeted therapy in acute myeloid leukemia. Adv Clin Exp Med. 2019;28(2):271-276. doi:10.17219/acem/81610

D0I

$10.17219 /$ acem $/ 81610$

\section{Copyright}

Copyright by Author(s)

This is an article distributed under the terms of the

Creative Commons Attribution Non-Commercial License

(http://creativecommons.org/licenses/by-nc-nd/4.0/) 
Acute myeloid leukemia (AML) occurs in 3-4 people out of 100,000 with a median age of 67 years. The 5 -year survival rate is $20 \%{ }^{1}$ The course of the disease depends on many factors, including cytogenetics, molecular genetics, comorbidity score, and the patient's age. Long-term survival rates for patients $<65$ years of age and $>65$ years of age are $40 \%$ and $5 \%$, respectively. ${ }^{2}$ Complete remission (CR) is achieved in $66 \%$ of elderly patients; in this group, the disease reoccurs in $16 \%$ of cases. ${ }^{3}$ Allogenic stem cell transplantation provides a chance for recovery from AML and longer overall survival (OS). The unsatisfactory results of previous AML treatment have encouraged the study of intracellular mechanisms that prolong the survival of leukemic cells and their resistance to apoptotic stimuli. These genetic changes have inspired the search for an effective targeted therapy. The first drug of this kind was all-trans retinoid acid (ATRA) in acute promyelocytic leukemia (APL), directed against the fusion of the genes $P M L$ and $R A R A$ caused by $\mathrm{t}(15 ; 17)$. The use of ATRA, especially with arsenic trioxide (ATO), has spectacularly improved OS and disease-free survival (DFS). ${ }^{4,5}$ Another turning point was the discovery of BCR-ABL kinase inhibitors. BCR-ABL kinase is formed by $t(9 ; 22)$, which is the most common mutation for chronic myeloid leukemia (CML), but which also occurs in acute myeloid leukemia (ALL) and in AML. Philadelphia chromosome-positive acute myeloid leukemia (AML Ph+) comprises $0.5-3 \%$ of AML cases.

The experiments conducted on leukemic cell lines, animal models and in clinical trials have led to the discovery of substances that can be classified according to their structure as having high or low molecular mass. ${ }^{6}$ The data is presented in Table 1.

\section{High molecular mass drugs}

Gemtuzumab ozogamicin (GO, Mylotarg) is an immunoconjugate compound created by the CD33 antibody, which is present on the surface of the myeloblasts in over 90\% of AML cases and is toxic to DNA calicheamicin. ${ }^{7}$ An epitope for GO, CD33 antigen, occurs in many expression and functional variants, and only some of these epitopes are sensitive to the cytotoxicity caused by GO. ${ }^{8}$ Despite GO withdrawal caused by toxicity in early clinical trials, subsequent trials have renewed the interest in this drug. In a meta-analysis of prospective phase III trials, it was proven that the use of GO in inductive therapy in variable age groups prolongs relapse-free survival (RFS) with tolerable adverse effects. Overall survival elongation thanks to GO was proven in most clinical trials in agedifferentiated groups, but the benefit for patients with adverse cytogenetics is controversial. Promising effects were observed among fit patients $>50$ years old and $>60$ years old not qualified for allogenic stem cell transplantation (alloSCT). The results of these trials suggest the advantage of using GO in bridge therapy before alloSCT with other
Table 1. Targeted drugs in AML treatment

\begin{tabular}{|c|c|c|c|}
\hline Target & Drug & \multicolumn{2}{|c|}{ Group } \\
\hline CD33 & $\begin{array}{c}\text { gemtuzumab } \\
\text { ozogamycin, } \\
\text { lintuzumab, } \\
\text { vadastuximab talirine }\end{array}$ & \multirow{2}{*}{\multicolumn{2}{|c|}{$\begin{array}{c}\text { high molecular mass } \\
\text { drugs }\end{array}$}} \\
\hline CD33, CD3 & AMG 330 & & \\
\hline FLT3 & $\begin{array}{l}1^{\text {st }} \text {-generation: } \\
\text { sorafenib, midostaurin, } \\
\text { lestaurtinib, sunitinib, } \\
\text { tandutinib, pacritinib; } \\
2^{\text {nd }} \text {-generation: } \\
\text { quizartinib, } \\
\text { crenolamid, ponatinib, } \\
\text { PLX3397, gliteritinib, } \\
\text { JH-IX-179 }\end{array}$ & \multirow[t]{5}{*}{$\begin{array}{l}\text { tyrosine } \\
\text { kinase } \\
\text { inhibitors }\end{array}$} & \multirow{16}{*}{$\begin{array}{c}\text { low } \\
\text { molecula } \\
\text { mass } \\
\text { drugs }\end{array}$} \\
\hline PLK1 & volasertib & & \\
\hline CDK & flavopiridol & & \\
\hline AURK & alisertib, barasertib & & \\
\hline PIM & AZD1208, SGI-1776 & & \\
\hline $\mathrm{IDH}$ & cenasidenib & \multirow{6}{*}{$\begin{array}{l}\text { cell pathway } \\
\text { inhibitors }\end{array}$} & \\
\hline GLI & GANT61 & & \\
\hline $\mathrm{BCL}-2$ & navitoclax, venetoclax & & \\
\hline NAE & pevonedistat & & \\
\hline topoisomerase II & vosaroxin & & \\
\hline BET & OTX015, ARV-825 & & \\
\hline LSD1 & ORY-1001, GSK2879552 & \multirow{5}{*}{$\begin{array}{l}\text { epigenetic } \\
\text { modulators }\end{array}$} & \\
\hline HDAC & pabinostat, vorinostat & & \\
\hline DOTL1L & pinometostat & & \\
\hline PD1/PDL1 & nivolumab & & \\
\hline MDM2 & RG7112, idasanutlin & & \\
\hline
\end{tabular}

FLT3 - FMS-like tyrosine kinase-3; PLK1 - polo-like kinase 1; CDK - cyclindependent kinases; AURK - aurora kinase; PIM - proviral insertion in + murine; IDH - isocitrate dehydrogenase; GLI - glioma; BCL-2 - B-cell lymphoma 2; NAE - NEDD8 activating enzyme; BET - bromodomain and extraterminal; LSD1 - lysine-specific demethylase; HDAC - histone deacetylase; PD1/PDL1 - programmed death-1/programmed death-1 ligand.

cytostatics. ${ }^{910}$ Promising results were achieved with GO applied in AML relapse after stem cell transplantation (SCT) therapy. On September 1, 2017, the U.S. Food and Drug Agency (FDA) approved GO for treatment in adults with newly diagnosed CD33+ AML.

BI 836858, lintuzumab (SGN-33; HuM195) and vadastuximab talirine are new anti-CD33 antibodies. Lintuzumab used with standard chemotherapy resulted in OS prolongation in a group of previously untreated patients who were unfit for intensive chemotherapy, aged 60-87 years, with an intermediate or adverse prognosis. ${ }^{11,12}$ The use of vadastuximab talirine is undergoing a phase III trial in a group of elderly patients.

AMG 330 (bispecific T-cell engager antibody [BiTE]) is a new antibody directed against both CD33 and CD3, which are present on the surface of $\mathrm{T}$ lymphocytes. Bispecific T-cell engager antibody was created to engage the cytotoxic response of $\mathrm{T}$ cells against leukemic cells 
in order to avert their immunological escape. ${ }^{13}$ It showed the best results among previously untreated AML patients with standard prognosis. In addition, tetravalent bispecific anti-CD33/CD3, bispecific anti-CD3 and C-type lectin-like molecule-1 (CCL-1), which can be found on most leukemic cells, are being investigated in animals. ${ }^{14}$

Ulocuplumab (BMS-936564/MDX-1338) is a monoclonal antibody which inhibits the binding of the CXC chemokine receptor 4 (CXCR4) to stimulate migration from the bone marrow to peripheral blood stromal cell-derived chemokine CXC motif ligand 12 (CXCL12). CXCR4 is overexpressed on AML blasts, among others. CXCR4 inhibition restricts AML cell growth and induces their apoptosis. In the first clinical trial on patients with relapsed/refractory AML, ulocuplumab in combination with mitoxantrone, etoposide and cytarabine led to CR with incomplete marrow recovery (CRi) in 51\% of the group of 73 patients. ${ }^{15}$ In December 2015, the FDA decided to use ulocuplumab as an orphan drug.

\section{Low molecular mass drugs}

The FMS-like tyrosine kinase-3 (FLT3) gene mutations FLT3-ITD and FLT3-TDK occur in 30\% and 7\% of AML cases, respectively. The FLT3 kinase inhibitors may be divided into $1^{\text {st }}$ - and $2^{\text {nd }}$-generation drugs. The first group $\left(1^{\text {st }}\right.$-generation $)$ are polykinase inhibitors, while the newer drugs are more selective molecules, which makes them safer and more effective. ${ }^{16}$ The mutated FLT3 gene has variable sensitivity to different drugs. ${ }^{17}$

Sorafenib is a multikinase inhibitor. It inhibits C-RAF, FLT3, VEGFR2, VEGFR3, and PDGFR family kinases. The action of sorafenib is amplified by the activation of p-AMPK by metformin, which potentiates the proapoptotic and antiproliferative effect. Glycolysis inhibition also plays a synergistic role, which has been proven in an animal model. ${ }^{18}$ According to the National Comprehensive Cancer Network (NCCN) guidelines, sorafenib is used in the treatment of refractory/recurrent AML, in monotherapy or with other drugs. Despite a higher response rate in FLT3+ patients than in FLT3- patients, sorafenib does not influence OS. In addition, it is not effective in elderly patients. When added to standard chemotherapy in patients younger than 60 years, sorafenib prolongs DFS. ${ }^{19}$ Moreover, sorafenib is effective before and after SCT it prolongs DFS and OS, maintains remission in sustained therapy after SCT in $100 \%$ of patients, and has a hematological response $>90 \%$ in AML recurrence after SCT. ${ }^{20-22}$ Promising effects have been reported from combining sorafenib with hypomethylating agents, ATRA or homoharringtonin, especially in refractory AML. ${ }^{23-25}$ The greatest antiproliferative and proapoptotic accuracy in preclinical trials on human leukemic cell lines was demonstrated with a composite of 3 kinase inhibitors: FLT3 (sunitinib), PI3K (PF-04691502) and GLI1/2 (GANT61). ${ }^{26}$
The use of sunitinib with standard inductive and life-sustaining therapy showed no benefits because of toxicity. The combination of FLT3 and AKT inhibitors is associated with the induction of resistance due to the protective effect of stroma on leukemia cells.

Lestaurtinib is a multikinase inhibitor whose targets include JAK-2. Added to a standard first-line FLT3+ AML therapy, it does not provide any benefits. ${ }^{27}$

Midostaurin used in monotherapy or in combination with different cytostatics in intensive chemotherapy has prolonged OS with tolerable toxicity in FLT3+ AML patients: studies include the addition of midostaurin in inductive or consolidative therapy, in sustaining therapy, in AML relapse, and in bridge therapy before SCT. ${ }^{28-31}$ The greatest benefits were observed in a group of patients who did not qualify for SCT previously untreated with FLT3 inhibitors. A promising effect was achieved in preclinical trials by a combination of ATRA and midostaurin due to the synergic effect against leukemic cells. Midostaurin combined with other drugs was registered by the FDA in the treatment of refractory/relapsed AML with the FLT3 mutation. ${ }^{32}$

Crenolamid represents a new, selective FLT3 kinase inhibitor group. It is currently in phase II trials. There is a possible synergy in using it with sorafenib against leukemic cells.

Quizartinib (AC220) has a high affinity for wild-type and mutated FLT3 kinase and has successfully completed phase I trials on a pediatric FLT3+ AML population. ${ }^{33}$ Its activity was demonstrated in refractory/relapse AML. AKN 028, a dose-dependent FLT3 kinase inhibitor that stops the cell cycle, is still being investigated. Gliteritinib is a selective FLT3/ASXL1 inhibitor. Used in a group of 80 patients with refractory/relapse FLT3+ AML, it resulted in a $55 \%$ response rate and it doubled OS. Gliteritinib is currently under investigation in supportive care and rescue therapy. Kinase inhibitors may generate secondary mutations. ${ }^{34}$ In trials on human leukemic cells, FLT3 mutations resistant to AC220 and sorafenib succumbed to a new kinase inhibitor, TT-3002. New molecules are being investigated, for example, AMG 925.

Volasertib (BI6727) is a polo-like kinase (PLK) inhibitor. Polo-like kinases play a key role in mitosis. There is higher PLK expression in AML, Hodgkin lymphoma (HL), nonsmall-cell lung cancer (NSCLC), and breast cancer, and its concentration correlates with mortality. ${ }^{35}$ In preclinical trials on leukemic cells acquired from patients, volasertib proved effective in monotherapy and with antimetabolites, hypomethylating agents and quizartinib. Associated with small doses of cytarabine, it increased CR and DFS in a previously untreated group of patients who, in the investigators' opinion, were unfit for intensive chemotherapy. In 2016, volasertib was called a breakthrough drug in the treatment of AML by the FDA. ${ }^{36}$

Flavopiridol (alvocidib) is a cyclin-dependent kinase (CDK), which induces cell cycle arrest and apoptosis 
in leukemic cells. The latest studies show no benefits over standard chemotherapy in previously untreated AML patients with an intermediate or adverse prognosis.

Alisertib (MLN8237) is an orally taken aurora kinase A (AurKA) inhibitor. Its synergy with cytarabine has been proven in preclinical tests. In phase II studies on a group of refractory/relapsed AML patients who did not qualify for standard chemotherapy, the disease was stabilized in nearly half of the patients.

Barasertib is an aurora kinase B (AurKB) inhibitor. It is more effective in prolonging OS in patients $>60$ years of age, but is more toxic in comparison with cytarabine. AZD1208 is an inhibitor of all proviral insertion in + murine (PIM) kinases, which in correlation with PIM1 expression inhibits the growth of 5 of the 14 AML cell lines, including FLT3-ITD+. The PIM kinase inhibitors show synergy with mTOR and AKT inhibitors in suppressing leukemic cells and in sensitizing AML cells to topoisomerase II inhibitors. ${ }^{37}$ SGI-1776 acts similarly; it has also been tested in NCL2 inhibition.

\section{Cell pathway inhibitors}

Isocitrate dehydrogenase (IDH) takes part in lipid metabolism and the Krebs cycle, and it catalyzes the transformation of isocitrate to $\alpha$-ketoglutarate. The IDH1 and IDH2 gene mutations occur in $11 \%$ and $12 \%$ of AML cases, respectively. Enasidenib (AG-221/CC-90007) is the first selective IDH2 inhibitor to induce the differentiation of leukemic cells. ${ }^{38-40}$ Enasidenib is taken orally and is active in monotherapy. It has been well-tolerated in phase II studies on patients with refractory/relapsed AML and has achieved an overall response rate (ORR) of $40 \%$ with a median response duration of 6 months. ${ }^{41}$ On August 1, 2017, enasidenib was approved by the FDA for the treatment of adult patients with relapsed/refractory AML with the IDH2 mutation.

The expression of the glioma (GLI) family transcription factors, which are the last part of the Hedgehog proliferative signal pathway, is a negative prognostic factor in AML. This finding has inspired the search for GLI inhibitors. ${ }^{42}$ The small-molecule inhibitor GANT61 is currently being studied. ${ }^{43}$

Navitoclax (ABT-263) is a BCL-2, BCL-XL and BCL-W protein family inhibitor. Its antitumor activity is restricted by adverse effects. Venetoclax (ABT-199) is a small-molecule antiapoptotic BCL-2 protein inhibitor which is registered by the FDA for treating chronic lymphocytic leukemia (CLL) and AML. ${ }^{44}$ In a high-risk recurrent/refractory AML patient group, a 38\% response rate was achieved, half of these being complete responses according to the International Working Group (IWG) criteria. ${ }^{45}$

There have been studies on molecules influencing the suppressor protein p53 pathway, for instance, the HDM2 inhibitor CGM097, which neutralizes the p53-inhibiting effect of HDM2 on AML cells.
Tosedostat is an aminopeptidase inhibitor that blocks the destruction and rebuilding of intercellular proteins. It is undergoing phase II trials. It was demonstrated on a group of patients $>60$ years of age with relapsed/refractory AML that tosedostat is active in monotherapy, doseindependently. In the same group with a negative prognosis, tosedostat combined with cytarabine and azacitidine achieved a 30\% ORR. Used on elderly patients in inductive therapy with cytarabine or decitabine, it resulted in a CR or CRi of more than $50 \%{ }^{46}$

Pevonedistat (MLN4924) is an NEDD8-activating enzyme (NAE) inhibitor that controls the destruction of many proteins taking part in the cell cycle, signal transduction, the destruction of DNA, or the stress response, for example, p53, p27, cyclin E, c-MYC, phospho-IkB $\alpha$, CDT-1, NRF-2, and HIF- $1 \alpha$. In preclinical tests, pevonedistat was effective in monotherapy in amplifying cytarabine action, but there was only a $20 \%$ response rate.

Vosaroxin is a topoisomerase II inhibitor which is essential for cell survival. Vosaroxin induces DNA destruction and is most effective among elderly patients diagnosed with AML or myelodysplastic syndrome (MDS). Phase III trials showed that vosaroxin prolongs survival by about 6 weeks. ${ }^{47}$

OTX015 is a BRD2/3/4 inhibitor indispensable for leukemic clone survival of c-MYC. OTX015 used in conjunction with pabinostat and azacitidine showed synergic activity towards KASUMI AML cell lines. ARV-825 was more effective against AML post-myeloproliferative cell lines than OTX015. Both drugs are in phase I trials. ${ }^{48}$

\section{Epigenetic modulators}

Lysine-specific demethylase 1 (LSD1) is a histone demethylase. ${ }^{49}$ Its expression has been demonstrated in many neoplasms and it plays a role in the self-renewal of AML stem cells. LSD1 inhibition leads to the inhibition of tumor growth and metastasis. ORY-1001 and GSK2879552 are tranylcypromine-derivative LSD1 inhibitors, both in phase I trials. ${ }^{50,51}$

Panobinostat (LBH589) induces AML cell apoptosis in vitro by inhibiting the expression of repair proteins (e.g., BRCA1, CHK1 and RAD51), increasing the efficiency of cytarabine and daunorubicin, and it is promising in $\mathrm{t}(8 ; 21) \mathrm{AML}$ due to the pathological AML1/ETO protein that recruits histone deacetylases. ${ }^{52}$

Vorinostat (suberoylanilidehydroxamic acid [SAHA]) promotes cell cycle inhibition and arrested growth, and induces differentiation and AML cell apoptosis. In phase II trials with cytarabine on AML/MDS patients with severe concomitant diseases, there was a median OS $>7$ months with acceptable toxicity. ${ }^{53}$

Histone deacetylase inhibitors, such as pracinostat and entinostat, are under investigation in AML patients. ${ }^{54,55}$

Rearranged mixed lineage leukemia (rMLL) is associated with an aggressive disease course and a poor response 
to multidrug chemotherapy, which is caused by a higher expression of HOXA9 and MEIS1. ${ }^{56}$ Pinometostat (EPZ$5676)$ is a histone methyltransferase DOT1L enzyme inhibitor. DOT1L is the rMLL target gene. Pinometostat is undergoing phase I trials. ${ }^{57}$

The programmed death-1 (PD-1) receptor occurs on activated $T$ cells and after binding with its programmed death 1 or 2 ligand (PDL-1, PDL-2), it suppresses T cell cytotoxic activity. This immunological escape has been presented in many cell lines, including AML. High PDL-1 expression correlates with an unfavorable course. Nivolumab is a PDL1 inhibitor which has been approved by the FDA for treating non-small-cell lung cancer, melanoma and renal cancer. Nivolumab with azacitidine is now in phase II studies in relapsed AML patients. A remission rate of $18 \%$ was achieved in elderly patients with tolerable side effects. ${ }^{58}$

The suppressor gene $p 53$ is called a genome warden due to its prevention of the replication of defective genome material and it leads to apoptosis. The destruction of the p53 protein is proceeded by ubiquitination after connecting with the MDM2 protein. A high MDM2 concentration with wild-type p53 appears in about $90 \%$ of AML types. RG 7112 is a $1^{\text {st }}$-generation MDM2 inhibitor. In phase I clinical studies, RG 7112 was effective in refractory/relapsed AML and in CLL. ${ }^{59}$

Idasanutlin (R7388) is a selective, next-generation MDM2 inhibitor. There were promising effects of phase I trials in refractory/relapsed AML: the higher the MDM2 expression was, the better the response to the drug was. ${ }^{60}$ Idasanutlin is better tolerated than RG 7112.

Despite the growing interest awakened by targeted therapy in AML treatment, the current results are unsatisfactory. Undoubtedly, this is due to the complexity of leukemogenic mechanisms. There is potential for further investigations and clinical studies to improve AML therapy. All in all, each study brings us closer to achieving success in AML therapy.

\section{References}

1. Kell J. Considerations and challenges for patients with refractory and relapsed acute myeloid leukaemia. Leuk Res. 2016;47:149-160. doi:10. 1016/j.leukres.2016.05.025

2. Almeida $A M$, Ramos F. Acute myeloid leukemia in the older adults. Leuk Res Rep. 2016;16(6):1-7. doi:10.1016/j.Irr.2016.06.001

3. von dem Borne PA, de Wreede LC, Halkes CJ, Marijt WA, Falkenburg $\mathrm{JH}$, Veelken $\mathrm{H}$. Effectivity of a strategy in elderly AML patients to reach allogeneic stem cell transplantation using intensive chemotherapy: Long-term survival is dependent on complete remission after first induction therapy. Leuk Res. 2016;46:45-50. doi:10.1016/j. leukres.2016.03.010

4. Kayser S, Krzykalla J, Elliott MA, et al. Characteristics and outcome of patients with therapy-related acute promyelocy tic leukemia frontline treated with or without arsenic trioxide. Leukemia. 2017;31(11): 2347-2354. doi:10.1038/leu.2017.92

5. Platzbecker U, Avvisati G, Cicconi L, et al. Improved outcomes with retinoic acid and arsenic trioxide compared with retinoic acid and chemotherapy in non-high-risk acute promyelocytic leukemia: Final results of the randomized Italian-German APL0406 trial. JClin Oncol. 2017;35(6):605-612. doi:10.1200/JCO.2016.67.1982
6. Hołowiecki J, Hołowiecka A. Targeted therapy in acute myeloid leukemia [in Polish]. Acta Haematol Pol. 2013;44(2):85-92.

7. De Witte T, Amadori S. The optimal dosing of gemtuzumab ozagamicin: Where to go from here? Haematologica. 2016;101(6):653-654. doi:10.3324/haematol.2016.145763

8. Laszlo GS, Harrington $\mathrm{KH}$, Gudgeon CJ, et al. Expression and functional characterization of CD33 transcript variants in human acute myeloid leukemia. Oncotarget. 2016;7(28):43281-43294. doi:10.18632/ oncotarget.9674

9. Paubelle E, Ducastelle-Leprêtre $\mathrm{S}$, Labussière-Wallet $\mathrm{H}$, et al. Fractionated gemtuzumab ozogamicin combined with intermediate-dose cytarabine and daunorubicin as salvage therapy in very high-risk AML patients: A bridge to reduced intensity conditioning transplant? Ann Hematol. 2017;96(3):363-371. doi:10.1007/s00277-016-2899-0

10. Zahler S, Bhatia M, Ricci A, et al. A phase I study of reduced-intensity conditioning and allogeneic stem cell transplantation followed by dose escalation of targeted consolidation immunotherapy with gemtuzumab ozogamicin in children and adolescents with CD33+ acute myeloid leukemia. Biol Blood Marrow Transplant. 2016;22(4): 698-704. doi:10.1016/j.bbmt.2016.01.019

11. Bixby DA, Fathi AT, Kovacsovics TJ, et al. Vadastuximab talirine monotherapy in older patients with treatment naive CD33-positive acute myeloid leukemia (AML) Blood. 2016;128(22):590.

12. Erba HP, Vasu S, Stein AS, et al. A phase $1 \mathrm{~b}$ study of vadastuximab talirine in combination with $7+3$ induction therapy for patients with newly diagnosed acute myeloid leukemia (AML). Blood. 2016;128 (22):211.

13. Masarova L, Kantarjian H, Garcia-Mannero G, Ravandi F, Sharma P, Daver N. Harnessing the immune system against leukemia: Monoclonal antibodies and checkpoint strategies for AML. Adv Exp Med Biol. 2017;995:73-95. doi:10.1007/978-3-319-53156-4_4

14. Leong SR, Sukumaran S, Hristopoulos M, et al. An anti-CD3/antiCLL-1 bispecific antibody for the treatment of acute myeloid leukemia. Blood. 2017;129(5):609-618. doi:10.1182/blood-2016-08-735365

15. Cho BS, Kim HJ, Konopleva M. Targeting the CXCL12/CXCR4 axis in acute myeloid leukemia: From bench to bedside. Korean JIntern Med. 2017;32(2):248-257. doi:10.3904/kjim.2016.244

16. Hassanein M, Almahayni MH, Ahmed SO, Gaballa S, El Fakih R. FLT3 inhibitors for treating acute myeloid leukemia. Clin Lymphoma Myeloma Leuk. 2016;16(10):543-549. doi:10.1016/j.clml.2016.06.002

17. Nguyen $B$, Williams AB, Young DJ, et al. FLT3 activating mutations display differential sensitivity to multiple tyrosine kinase inhibitors. Oncotarget. 2017;8(7):10931-10944. doi:10.18632/oncotarget.14539

18. Ju HQ, Zhan G, Huang A, et al. ITD mutation in FLT3 tyrosine kinase promotes Warburg effect and renders therapeutic sensitivity to glycolytic inhibition. Leukemia. 2017;31(10):2143-2150. doi:10.1038/leu. 2017.45

19. Roolf C, Dybowski N, Sekora A, et al. Phosphoproteome analysis reveals differential mode of action of sorafenib in wildtype and mutated FLT3 AML cells. Mol Cell Proteomics. 2017;16(7):1365-1376. doi:10.1074/mcp.M117.067462

20. Battipaglia G, Ruggeri A, Massoud R, et al. Efficacy and feasibility of sorafenib as a maintenance agent after allogeneic hematopoietic stem cell transplantation for Fms-like tyrosine kinase 3-mutated acute myeloid leukemia. Cancer. 2017;123(15):2867-2874. doi:10.1002/ cncr.30680

21. De Freitas T, Marktel S, Piemontese $S$, et al. High rate of hematological responses to sorafenib in FLT3-ITD acute myeloid leukemia relapsed after allogeneic hematopoietic stem cell transplantation. Eur J Haematol. 2016;96(6):629-636. doi:10.1111/ejh.12647

22. Brunner AM, Li S, Fathi AT, et al. Haematopoietic cell transplantation with and without sorafenib maintenance for patients with FLT3-ITD acute myeloid leukaemia in first complete remission. $\mathrm{Br} J$ Haematol. 2016;175(3):496-504. doi:10.1111/bjh.14260

23. Ernst J, Schäfer V, Rinke J, et al. Continuous molecular remission and regression of side effects after discontinuation of salvage therapy with sorafenib and donor lymphocyte infusions in a young patient with relapsed AML. Ann Hematol. 2016;95(6):1027-1030. doi:10.1007/ s00277-016-2637-7

24. Wang R, Xia L, Gabrilove J, Waxman S, Jing Y. Sorafenib inhibition of Mcl-1 accelerates ATRA-induced apoptosis in differentiation-responsive AML cells. Clin Cancer Res. 2016;22(5):1211-1221. doi:10.1158/10780432.CCR-15-0663 
25. Lam SS, Ho ES, He BL, et al. Homoharringtonine (omacetaxine mepesuccinate) as an adjunct for FLT3-ITD acute myeloid leukemia. SCi Transl Med. 2016;8(359):359ra129.

26. Latuske EM, Stamm H, Klokow M, et al. Combined inhibition of GLI and FLT3 signaling leads to effective anti-leukemic effects in human acute myeloid leukemia. Oncotarget. 2017;8(17):29187-29201. doi:10. 18632/oncotarget.16304

27. Knapper S, Russell N, Gilkes A, et al. A randomized assessment of adding the kinase inhibitor lestaurtinib to first-line chemotherapy for FLT3-mutated AML. Blood. 2017;129(9):1143-1154. doi:10.1182/blood2016-07-730648

28. Gallogly MM, Lazarus HM. Midostaurin: An emerging treatment for acute myeloid leukemia patients. J Blood Med. 2016;7:73-83. doi:10. 2147/JBM.S100283

29. Starr P. Midostaurin the first targeted therapy to improve survival in AML: Potentially practice-changing. Am Health Drug Benefits. 2016; 9(Spec Issue):1-21.

30. Mazzarella L. Orlando Magic: Report from the $57^{\text {th }}$ meeting of the American Society of Haematology, 5-7 December 2015, Orlando, USA. Ecancermedicalscience. 2016;10:612. doi:10.3332/ecancer. 2016.612.

31. Smuga-Otto K. Midostaurin + chemo ups AML survival. CancerDiscov. 2016;6(2):OF2. doi:10.1158/2159-8290.CD-NB2015-177

32. Levis M. Midostaurin approved for FLT3-mutated AML. Blood. 2017; 129(26):3403-3406. doi:10.1182/blood-2017-05-782292

33. Cooper TM, Cassar J, Eckroth E, et al. A phase I study of quizartinib combined with chemotherapy in relapsed childhood leukemia: A Therapeutic Advances in Childhood Leukemia \& Lymphoma (TACL) Study. Clin Cancer Res. 2016;22(16):4014-4022. doi:10.1158/1078-0432. CCR-15-1998

34. Kasi PM, Litzow MR, Patnaik MM, Hashmi SK, Gangat N. Clonal evolution of AML on novel FMS-like tyrosine kinase-3 (FLT3) inhibitor therapy with evolving actionable targets. Leuk Res Rep. 2016;5:7-10. doi:10.1016/j.Irr.2016.01.002

35. Talati C, Griffiths EA, Wetzler M, Wang ES. Polo-like kinase inhibitors in hematologic malignancies. Crit Rev Oncol Hematol. 2016;98: 200-210. doi:10.1016/j.critrevonc.2015.10.013

36. van den Bossche J, Lardon F, Deschoolmeester V, et al. Spotlight on volasertib: Preclinical and clinical evaluation of a promising Plk1 inhibitor. Med Res Rev. 2016;36(4):749-786. doi:10.1002/med.21392

37. Doshi KA, Trotta R, Natarajan K, et al. Pim kinase inhibition sensitizes FLT3-ITD acute myeloid leukemia cells to topoisomerase 2 inhibitors through increased DNA damage and oxidative stress. Oncotarget. 2016;7(30):48280-48295. doi:10.18632/oncotarget.10209

38. Amatangelo MD, Quek L, Shih A, et al. Enasidenib induces acute myeloid leukemia cell differentiation to promote clinical response. Blood. 2017;130(6):732-741. doi:10.1182/blood-2017-04-779447

39. Travins J, Wang F, David MD, et al. AG-221, a first-in-class therapy targeting acute myeloid leukemia harboring oncogenic IDH2 mutations. Cancer Discov. 2017;7(5):478-493. doi:10.1158/2159-8290.CD-16-1034

40. Kats LM, Vervoort SJ, Cole R, et al. A pharmacogenomic approach validates AG-221 as an effective and on-target therapy in IDH2 mutant AML. Leukemia. 2017;31(6):1466-1470. doi:10.1038/leu.2017.84

41. Stein EM, DiNardo CD, Pollyea DA, et al. Enasidenib in mutant-IDH2 relapsed or refractory acute myeloid leukemia. Blood. 2017;130(6): 722-731. doi:10.1182/blood-2017-04-779405

42. Long B, Wang LX, Zheng FM, et al. Targeting GLI1 suppresses cell growth and enhances chemosensitivity in CD34+ enriched acute myeloid leukemia progenitor cells. Cell Physiol Biochem. 2016;38(4): 1288-1302. doi:10.1159/000443075

43. Masetti R, Bertuccio $S N$, Astolfi $A$, et al. Hh/Gli antagonist in acute myeloid leukemia with CBFA2T3-GLIS2 fusion gene. J Hematol Oncol. 2017;10(1):26. doi:10.1186/s13045-017-0396-0

44. Soderquist R, Eastman A. BCL2 Inhibitors as anticancer drugs: A plethora of misleading BH3 mimetics. Mol CancerTher. 2016;15(9):2011-2017. doi:10.1158/1535-7163.MCT-16-0031
45. Ruvolo PP, Ruvolo VR, Benton CB, et al. Combination of galectin inhibitor GCS-100 and BH3 mimetics eliminates both p53 wild type and p53 null AML cells. Biochim Biophys Acta. 2016;1863(4):562-571. doi:10. 1016/j.bbamcr.2015.12.008

46. Mawad R, Becker PS, Hendrie P, et al. Phase II study of tosedostat with cytarabine or decitabine in newly diagnosed older patients with acute myeloid leukaemia or high-risk MDS. Br J Haematol. 2016;172(2): 238-245. doi:10.1111/bjh.13829

47. Jamieson GC, Fox JA, Poi M, Strickland SA. Molecular and pharmacologic properties of the anticancer quinolone derivative vosaroxin: A new therapeutic agent for acute myeloid leukemia. Drugs. 2016; 76(13):1245-1255. doi:10.1007/s40265-016-0614-z

48. Saenz DT, Fiskus W, Qian Y, et al. Novel BET protein proteolysis-targeting chimera exerts superior lethal activity than bromodomain inhibitor (BETi) against post-myeloproliferative neoplasm secondary (s) AML cells. Leukemia. 2017;31(9):1951-1961. doi:10.1038/leu.2016.393

49. Maiques-Diaz A, Somervaille TC. LSD1: Biologic roles and therapeutic targeting. Epigenomics. 2016;8(8):1103-1116. doi:10.2217/epi-20160009

50. Zheng YC, Yu B, Jiang GZ, et al. Irreversible LSD1 inhibitors: Application of tranylcypromine and its derivatives in cancer treatment. Curr Top Med Chem. 2016;16(19):2179-2188.

51. Przespolewski A, Wang ES. Inhibitors of LSD1 as a potential therapy for acute myeloid leukemia. Expert Opin Investig Drugs. 2016;25(7): 771-780. doi:10.1080/13543784.2016.1175432

52. Zhao J, Xie C, Edwards H, Wang G, Taub JW, Ge Y. Histone deacetylases 1 and 2 cooperate in regulating BRCA1, CHK1, and RAD51 expression in acute myeloid leukemia cells. Oncotarget. 2017;8(4):6319-6329. doi:10.18632/oncotarget.14062

53. Montalban-Bravo G, Huang X, Jabbour E, et al. A clinical trial for patients with acute myeloid leukemia or myelodysplastic syndromes not eligible for standard clinical trials. Leukemia. 2017;31(2):318-324. doi:10.1038/leu.2016.303

54. Norsworthy KJ, Cho E, Arora J, et al. Differentiation therapy in poor risk myeloid malignancies: Results of companion phase II studies. Leuk Res. 2016;49:90-97. doi:10.1016/j.leukres.2016.09.003

55. Prebet T, Sun Z, Ketterling RP, et al.; Eastern Cooperative Oncology Group and North American Leukemia intergroup. Azacitidine with or without Entinostat for the treatment of therapy-related myeloid neoplasm: Further results of the E1905 North American Leukemia Intergroup study. Br J Haematol. 2016;172(3):384-391. doi:10.1111/ bjh.13832

56. Waters NJ. Preclinical pharmacokinetics and pharmacodynamics of pinometostat (EPZ-5676), a first-in-class, small molecule s-adenosyl methionine competitive inhibitor of DOT1L. Eur J Drug Metab Pharmacokinet. 2017;42(6):891-901. doi:10.1007/s13318-017-0404-3

57. Smith SA, Gagnon S, Waters NJ. Mechanistic investigations into the species differences in pinometostat clearance: Impact of binding to alpha-1-acid glycoprotein and permeability-limited hepatic uptake. Xenobiotica. 2017;47(3):185-193. doi:10.3109/00498254.2016.1173265

58. Daver N, Basu S, Garcia-Manero G, et al. Phase lb/ll study of nivolumab in combination with azacitidine (AZA) in patients ( $p t s)$ with relapsed acute myeloid leukemia (AML). Abstract \#763. Presented at the ASH Annual Meeting and Exhibition, December 6, 2016; San Diego, CA.

59. Andreeff M, Kelly KR, Yee K, et al. Results of the phase I trial of RG 7112, a small-molecule MDM2 antagonist in leukemia. Clin Cancer Res. 2016; 22(4):868-876. doi:10.1158/1078-0432.CCR-15-0481

60. Reis B, Jukofsky L, Chen G, et al. Acute myeloid leukemia patients' clinical response to idasanutlin (RG7388) is associated with pre-treatment MDM2 protein expression in leukemic blasts. Haematologica. 2016;101(5):e185-188. doi:10.3324/haematol.2015.139717 